\title{
Selection of the Optimal Spectral Resolution for the Cadmium-Lead Cross Contamination Diagnosing Based on the Hyperspectral Reflectance of Rice Canopy
}

\author{
Shuangyin Zhang ${ }^{1}{ }^{(}$, Ying Zhu ${ }^{1, *} \mathbb{C}$, Mi Wang ${ }^{1, *}$ and Teng Fei ${ }^{2}$ \\ 1 State Key Laboratory of Information Engineering in Surveying, Mapping and Remote Sensing, \\ Wuhan University, Wuhan 430079, China \\ 2 School of Resource and Environmental Sciences, Wuhan University, Wuhan 430079, China \\ * Correspondence: yzhu1003@whu.edu.cn (Y.Z.); wangmi@whu.edu.cn (M.W.); \\ Tel.: +86-138-7115-3292 (Y.Z.); +86-139-7135-1686 (M.W.)
}

Received: 15 August 2019; Accepted: 7 September 2019; Published: 9 September 2019

\begin{abstract}
This paper proposed an optimal spectral resolution for diagnosing cadmium-lead $(\mathrm{Cd}-\mathrm{Pb})$ cross contamination with different pollution levels based on the hyperspectral reflectance of rice canopy. Feature bands were sequentially selected by two-way analysis of variance (ANOVA2) and random forests from the high-dimensional hyperspectral data after preprocessing. Then Support Vector Machine (SVM) was applied to diagnose the pollution levels using different feature bands combination with different spectral resolutions and cross validation was conducted to evaluate the distinguishing accuracies. Finally, the optimal spectral resolution could be determined by comparing the diagnosing accuracies of the optimal feature bands combination in each spectral resolution. In the experiments, the hyperspectral reflectance data of rice canopy with ten different spectral resolutions was captured, covering 16 pretreatments of $\mathrm{Cd}$ and $\mathrm{Pb}$ pollution. The experimental results showed the optimal spectral resolution was $9 \mathrm{~nm}$ with the highest average accuracy of 0.71 and relatively standard deviation of 0.07 for diagnosing the categories and levels of $\mathrm{Cd}-\mathrm{Pb}$ cross contamination. The useful exploration provided an evidence for optimal spectral resolution selection to reduce the cost of heavy metal pollution diagnose.
\end{abstract}

Keywords: heavy metal pollution diagnosis; hyperspectral remote sensing; optimal spectral resolution selection; cross contamination

\section{Introduction}

Heavy metal pollution is seriously jeopardizing food security, and this hazard is continuing to intensify due to the increasing byproducts of frequent anthropogenic activities, such as industrial pollutants, wastewater, mining wastes, and pesticides [1,2]. Traditional methods for heavy metal diagnosis rely on wet chemistry analysis of a collection of soil samples, which has low-efficiency and is time-consuming [3,4], so high-efficiency and time-saving visible-near infrared reflectance spectroscopy (VNIRS) has become an alternative technology for diagnosing heavy metal pollutions.

Some investigations have shown the feasibility of VNIRS based methods to diagnose the polluted or stressed categories the plants suffer, including pest stress, salinity stress, water stress, and heavy metal pollution [5-13]. These studies explored its diagnostic ability on the basis of soil samples hyperspectral datasets measured by VNIRS in the laboratory. For non-heavy metal diagnosis, Moshou et al. and Huang et al. $[5,14]$ explored the feasibility of using the spectral reflectance of wheat to detect pest stress. Wang et al. [6] explored the possibility of interpreting water stress from plants using VNIRS. 
Tilley et al. and Rud et al. [7,15] investigated the feasibility of distinguishing salinity stress from plants using VNIRS. For heavy metals pollution diagnosis, some researchers have also explored the feasibility of diagnosing heavy metals based on VNIRS. Shi et al. [8] systematically explored the mechanisms of predicted concentrations, acquisition methods, preprocessing technologies, and modeling strategies. Liu et al. [9] investigated the feasibility of estimating heavy metal contaminations in floodplain soils. Similar to the Liu et al. [9], St. Luce et al. [16] explored the feasibility of predicting heavy metal concentrations based on visible near-infrared reflectance spectroscopy. Choe et al. [17] mapped heavy metal pollution in stream sediments by combining geochemistry, field spectroscopy, and hyperspectral remote sensing. Wang et al. [18] used VNIRS to predict low $\mathrm{Pb}$ concentrations and further investigated the predictive mechanism of heavy metal concentrations. Liu et al. [19] used VNIRS to try to monitor stress levels of rice with heavy metal pollution in a rice canopy, and recommended a fractal dimension of reflectance with a wavelet transform of $480-850 \mathrm{~nm}$ as a comprehensive indicator. Chen et al. [10] performed VINRS of 100 samples to rapidly identify the pollution risk of cadmium and identification of pollution hotspots was achieved by interpolating the predicted values. Shi et al. [11] even considered improving the diagnostic and predicted accuracies using combined VNIRS of rice plants and their soil.

However, these above studies only used a single spectral resolution with $1 \mathrm{~nm}$ for heavy metal pollution diagnosing, thus there was a lack of the exploration of the diagnostic ability with the different resolutions. The exploration does help to find an optimal spectral resolution for heavy metal pollution diagnosing, especially for cross contamination diagnosing. Some researches [20-22] have indicated that the higher spectral resolution not only increases the spectrometer production cost, but also involves diagnostic accuracy due to Hughes phenomenon [23]. So, it is necessary to explore the optimal spectral resolution for heavy metal cross contamination diagnosing.

Given the importance of optimal spectral resolution, this study aimed to propose a method to determinate the optimal spectral resolution for heavy metal cross contamination diagnosing in agricultural soils. To achieve this goal, the specific objectives were: (1) to design a cross contamination experiment with $\mathrm{Cd}$ and $\mathrm{Pb}$ with four different pollution concentrations; (2) acquire the hyperspectral reflectance of each sample at spectral resolutions; (3) select the number and combination of feature bands for subsequent diagnosis based on two-way analysis of variance (ANOVA2) and random forest (RF); and (4) determine the optimal spectral resolution for $\mathrm{Cd}-\mathrm{Pb}$ pollution diagnosing based on diagnostic accuracies of SVM. The result of this study is expected to give a suggestion for sensors' spectral resolution selection in heavy metal cross contamination diagnosing by using plant reflectance spectroscopy.

\section{Materials and Methods}

\subsection{Materials}

Rice, the dominant staple food in China [18], was used as an indicator for cross contamination diagnosing. $\mathrm{Pb}$ was the most seriously polluted source for rice in some southern provinces of China, followed by $\mathrm{Cd}$ [24], so $\mathrm{Cd}$ and $\mathrm{Pb}$ were selected for the pollution sources for cross contamination in this experiment. As opposed to soil cultures, water was used to plant rice to ensure the uniformity of the contaminative concentration. To avoid the influence of environmental factors, the containers were previously painted black [25]. Four different contaminative concentrations of Cd pollution, $0 \mathrm{mg} / \mathrm{L}$, $2 \mathrm{mg} / \mathrm{L}, 5 \mathrm{mg} / \mathrm{L}$, and $8 \mathrm{mg} / \mathrm{L}$, were used, while the concentrations of $\mathrm{Pb}$ pollution were $0 \mathrm{mg} / \mathrm{L}, 50 \mathrm{mg} / \mathrm{L}$, $100 \mathrm{mg} / \mathrm{L}$, and $500 \mathrm{mg} / \mathrm{L}$. Then, each Cd contaminative concentration as combined with that $\mathrm{of} \mathrm{Pb}$. Including the normal group, there were totally 16 groups in the experiment. The details of pollution pretreatments of the different groups are displayed in Table 1 . In the paper, $\mathrm{ZCd} / \mathrm{ZPb}, \mathrm{LCd} / \mathrm{LPb}$, $\mathrm{MCd} / \mathrm{MPb}$, and $\mathrm{HCd} / \mathrm{HPb}$ represented zero, low-, medium-, and high-level concentrations of $\mathrm{Cd} / \mathrm{Pb}$ pollution, respectively. The contaminative concentrations were determined by early pre-test after referring to the Risk Control Standard for Soil Contamination of Agricultural Land (GB15618-2008) of China [26] and previous published papers $[9,10,18,27-30]$. These concentrations were also closed to the 
controlled value in (GB15618-2008) - $3 \mathrm{mg} / \mathrm{kg}$ and $700 \mathrm{mg} / \mathrm{kg}$ for $\mathrm{Cd}$ and Pb respectively in farmland soil [26].

Table 1. Pollution pretreatments of different groups.

\begin{tabular}{cccc}
\hline Group Name & Pollution Pretreatment & Group Name & Pollution Pretreatment \\
\hline G01 & ZCd-ZPb & G09 & LCd-MPb \\
G02 & LCd-ZPb & G10 & LCd-HPb \\
$\mathrm{G} 03$ & $\mathrm{MCd}-\mathrm{ZPb}$ & $\mathrm{G} 11$ & $\mathrm{MCd}-\mathrm{LPb}$ \\
$\mathrm{G} 04$ & $\mathrm{HCd}-\mathrm{ZPb}$ & $\mathrm{G} 12$ & $\mathrm{MCd}-\mathrm{MPb}$ \\
$\mathrm{G} 05$ & $\mathrm{ZCd}-\mathrm{LPb}$ & $\mathrm{G} 13$ & $\mathrm{MCd}-\mathrm{HPb}$ \\
$\mathrm{G} 06$ & $\mathrm{G} 14$ & $\mathrm{HCd}-\mathrm{LPb}$ \\
$\mathrm{G} 07$ & $\mathrm{ZCd}-\mathrm{MPb}$ & $\mathrm{G} 15$ & $\mathrm{HCd}-\mathrm{MPb}$ \\
$\mathrm{G} 08$ & $\mathrm{ZCd}-\mathrm{HPb}$ & $\mathrm{G} 16$ & $\mathrm{HCd}-\mathrm{HPb}$ \\
\hline
\end{tabular}

An ASD FieldSpec®3 portable spectrometer (ASD Inc., now PANalytical Company, Boulder, CO, USA) with a spectral range of 350-2500 nm was used to measure the raw spectrum, and this equipment performed data collection with 10 scans per second [31,32]. The spectral measurements were conducted on a cloudless, sunny day between 10:00 am and 2:00 pm. For the measurements of each pollution pretreatment group, a standardized plate with $100 \%$ reflectance was used to calibrate the reflectance measurement [33]. To ensure the reliability of the measured spectrum, each sample underwent ten parallel measurements, with the mean value used as the final determination of the hyperspectral information. After screening, the spectral datasets, including six times complete measurements, were selected from multiple datasets for subsequent diagnostic research.

Based on the raw reflectance with a $1 \mathrm{~nm}$ spectral resolution, we used average to resample to different spectral resolution, and the subsequent diagnostic accuracies were also based on the spectral average. This study acquired ten kinds of hyperspectral dataset with the different resolution from $1 \mathrm{~nm}$ to $10 \mathrm{~nm}$.

\subsection{Methods}

To acquire the optimal spectral resolution for $\mathrm{Cd}-\mathrm{Pb}$ cross contamination diagnosing, the hyperspectral dataset with different band combinations and spectral resolutions were input the diagnostic model by preprocessing and selection, followed by the accuracy's comparison. This method mainly included four parts: (1) the hyperspectral preprocessing, (2) feature bands selection, (3) Cd and $\mathrm{Pb}$ diagnosing and accuracy evaluation, and (4) diagnosing accuracy comparison with different bands and spectral resolutions. The technological workflow is shown as Figure 1.

\subsubsection{Hyperspectral Data Preprocessing}

Due to instrument noise, the spectral bands (1351-1440 nm, 1801-2030 nm, and 2351-2500 nm) were removed to improve the signal-to-noise ratio. The representative curves of remained raw reflectance were shown in Figure 2. To extract differences and eliminate redundancy, the following preprocessing methods were performed for the raw hyperspectral data. The pretreatment of the differentials [34], including the first differential and second differential, reduced the interference of background noise. Savitzky and Golay smoothing [35] eliminated random noise. Normalization or standardization reduced information redundancy and extracted the difference. In practice, the combination of first derivative and normalization and the combination of second derivative and Savitzky and Golay smoothing were used to select the bands that were sensitive to Cd pollution and $\mathrm{Pb}$ pollution, respectively. 


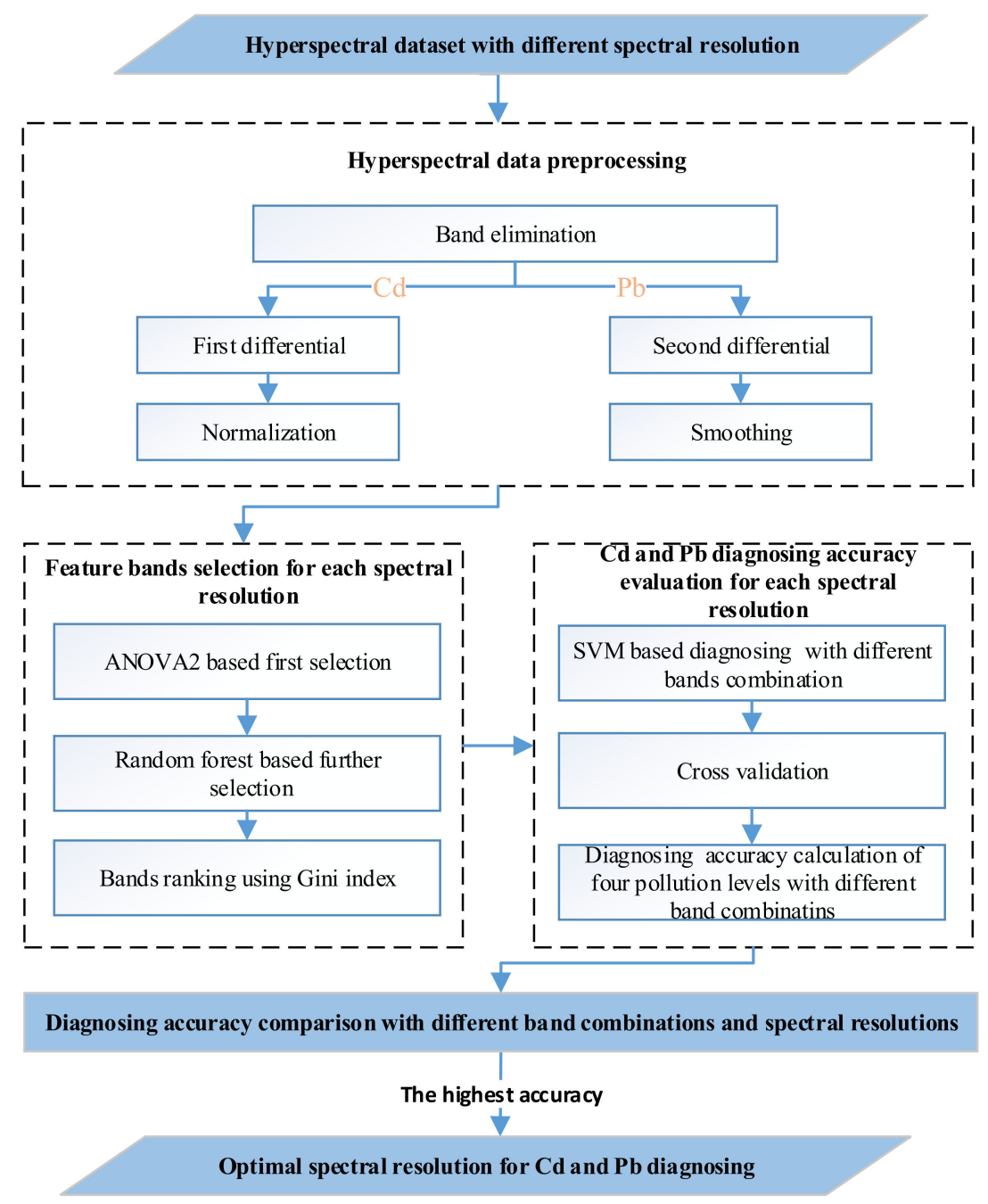

Figure 1. Workflow of the method.

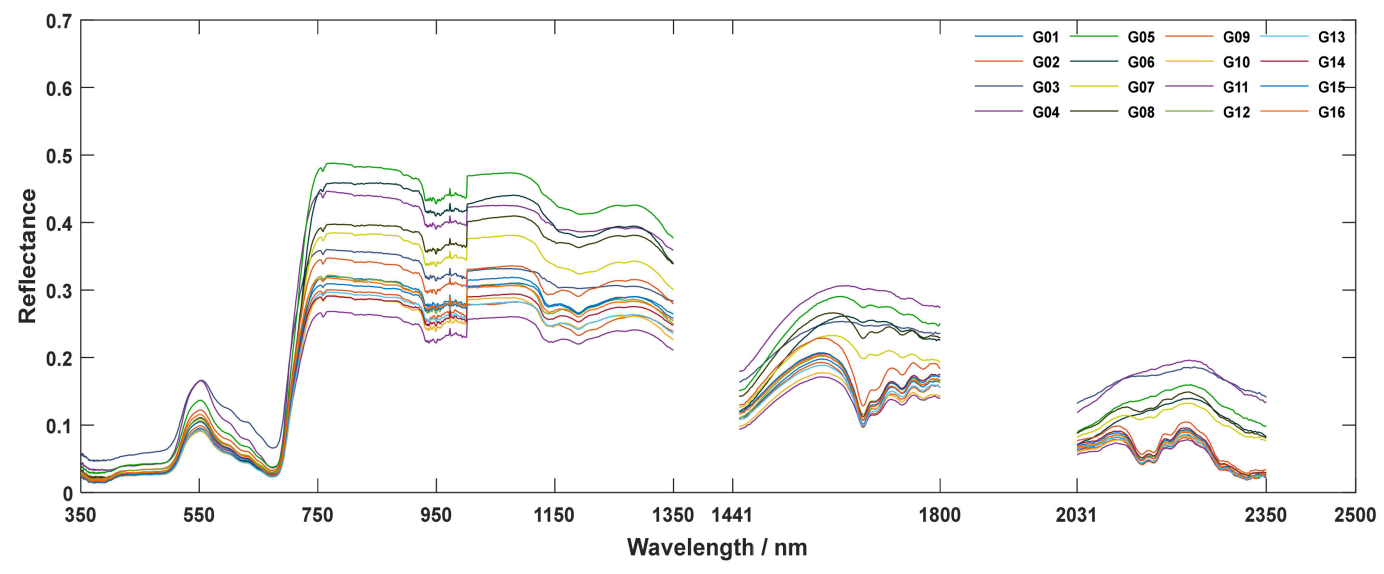

Figure 2. Raw reflectance after removing part of bands for first time measurement.

\subsubsection{Feature Bands Selection}

The number of input feature bands was one of the key factors for subsequent diagnosis. ANOVA2 and RF were used to determine the number. The three main steps of this analysis were as follows.

ANOVA2 was a statistical analysis method that could be used to analyze whether different levels of two pollutions had a significant impact on the polluted results and distinguished whether there was an interaction between the two pollutions based on the difference significances. Due to the uncertain 
inducement for interactions effects, ANOVA2 preferentially removed bands that were sensitive to interaction effects, followed by the feature bands first time selection. These feature bands that were sensitive to each of $\mathrm{Cd}$ and $\mathrm{Pb}$ pollutions were selected with less significance than preset value $(0.05)$.

$\mathrm{RF}$ was used to rank the feature bands based on the Gini index and further reduced to ten bands if the bands were more than ten, or all bands were reserved when the aforementioned bands did not reach ten. The Gini index was used as an evaluation indicator to measure the contribution rate of each feature band. The formula for Gini index displayed as following:

$$
G I_{m}=\sum_{k=1}^{|K|} \sum_{k \neq k} p_{m k} p_{m k}=1-\sum_{k=1}^{|K|} p_{m k}^{2}
$$

where $K$ is the number of the pollution concentration, and its value is four in the study, $p_{m k}$ represents the proportion of the pollution concentration $K$ in the node $m$. In this experiment, the 0.75 dataset was randomly selected as training data, and the remainder was used for validation datasets. Random forest runs hundreds of times to eliminate the randomness of feature band selection. In each operation, the band with the highest Gini index was reserved, and the feature band ranked on the basis of their frequencies that Gini index was top one. Through testing, we found the feature band importance of each band was stable when the program ran 1000 times repeatedly.

RF ranked the order of the bands based on the Gini index. However, RF did not determine the number of band combinations, an important factor for determining subsequent diagnostic accuracy. The overall diagnostic results of four different contaminative concentrations took the four overall accuracies into consideration, resulting in a relatively sound value, so the overall diagnostic results were used to determine the number of feature bands for each spectral resolution. For each spectral resolution, the diagnostic results were achieved by subsequent SVM model and cross validation with increasing the number of input bands.

\subsection{3. $\mathrm{Cd}$ and $\mathrm{Pb}$ Diagnosing and Accuracy Evaluation}

SVM was used as diagnostic models to distinguish the polluted categories and levels. The SVM converted linearly indivisible low-dimensional data into high-dimensional data, making it linearly separable by finding the optimal hyperplane, which was suitable for diagnosing and classifying the study with small samples. All of the programs ran in the Matlab 2015a platform. Through testing, the default settings for the SVM model with the linear kernel function reached the relatively optimal setting values. For any spectral resolution, the SVM model run repeatedly to diagnose the special categories and levels with the increase of the number of input bands from one to ten.

The leave-one-out cross-validation (LOOCV) were used to evaluate the diagnostic model performance. Different from dividing the training dataset and the verification dataset proportionally, the LOOCV method made full use of all hyperspectral data, which eliminated the randomness of diagnosing small data samples. Based on the preset classification label, one data was reserved for verification dataset each time, and the rest were used as training dataset. Finally, the average value of all verification results was used as the accuracy of the diagnostic model.

\subsubsection{Diagnosing Accuracy Comparison with Different Bands and Spectral Resolutions}

The part aimed at selecting a spectral resolution that was applicable to diagnose a kind of $\mathrm{Cd}$ and $\mathrm{Pb}$ pollution no matter how the levels changed. The average accuracy of different levels was used as an indicator to determine the optimal bands and the optimal resolution. The highest average accuracy firstly determined the optimal bands combination under a single spectral resolution, and then determined the optimal spectral resolution. The optimal bands and the optimal resolution were determined

$$
\text { The optimal bands }= \begin{cases}\text { the highest } A V & \text { only top value } \\ \text { the highest } A V \text { with less band number } & \text { two or more top value }\end{cases}
$$


The optimal resolution $= \begin{cases}\text { the highest AV } & \text { only top value } \\ \text { the highest AV with less coarser resolution } & \text { two or more top value }\end{cases}$

with average accuracy (AV) of different pollution levels.

\section{Results}

\subsection{Results of Feature Bands Selection}

The feature bands were selected by ANOVA2, followed by the random forest algorithm. Before preprocessing, there were 1660 bands. ANOVA2 greatly reduced the dimensions of hyperspectral data by an order of magnitude. The number of sensitive bands of $\mathrm{Cd}$ was greater than that of $\mathrm{Pb}$ with the exception at the $2 \mathrm{~nm}$ resolution.

Figures 3 and 4 showed the changes of the highest accuracies with the increase the number of input bands from one to ten. The $x$-axis represented the number of input bands, and the $y$-axis represented the spectral resolution.

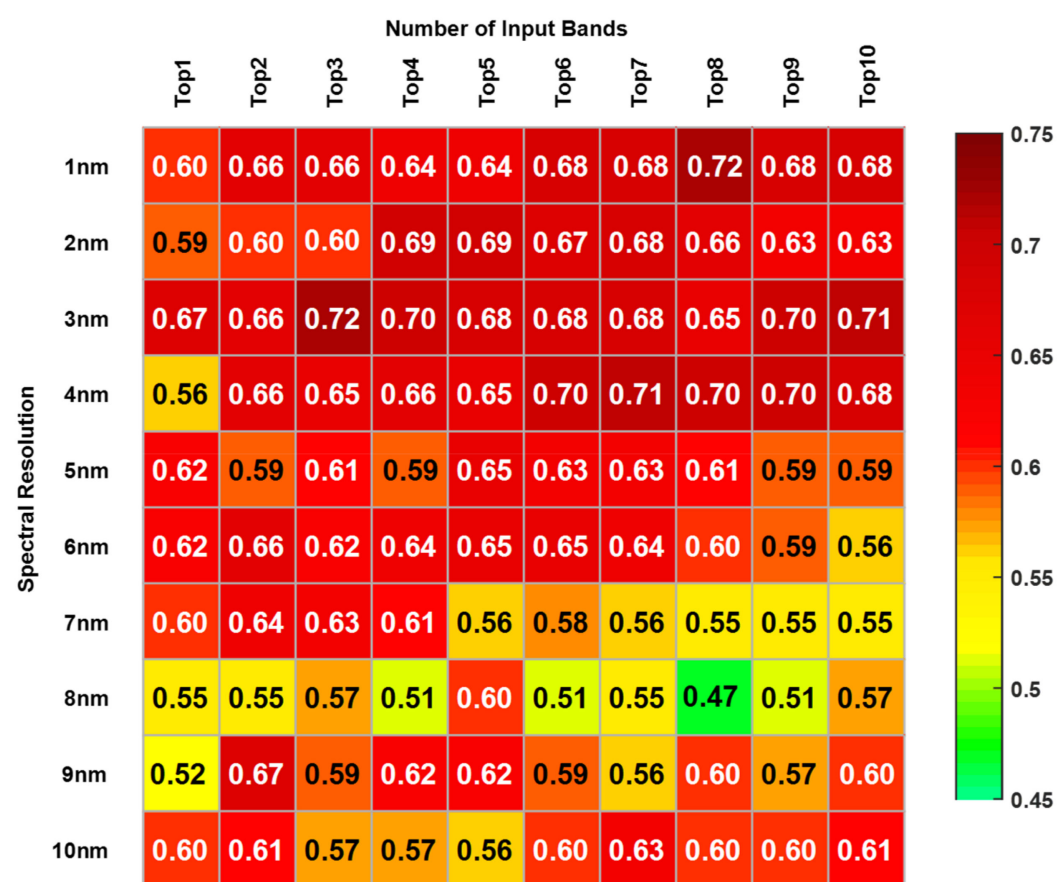

Figure 3. Diagnostic accuracies of Cd pollution diagnosing for band combination selecting.

As shown in Figure 3, the average accuracies for $\mathrm{Cd}$ pollution were distributed in the range from 0.51 to 0.72 except the $0.47 \mathrm{in} 8 \mathrm{~nm}$ resolution. As the input band changed, the relative highest accuracies of each resolution ranged from 0.60 to 0.72 , with a maximum of 0.72 at a $1 \mathrm{~nm}$ spectral resolution with eight input bands and $3 \mathrm{~nm}$ spectral resolution with three input bands. As the resolution changed, the maximum value of each resolution tended to decrease overall. There was a stable value at $3 \mathrm{~nm}$, and all accuracies were not less 0.65 , no matter how the number of input bands changed.

The average accuracies for $\mathrm{Pb}$ pollution ranged from 0.45 to 0.75 , but nearly half of the highest accuracies were above 0.60 , as shown in Figure 4 . The accuracies at $1 \mathrm{~nm}$ and $2 \mathrm{~nm}$ had a distinct advantage, displaying a saffron yellow color. The accuracies at $3 \mathrm{~nm}$ were generally low, and the lowest at $10 \mathrm{~nm}$. The highest accuracy of ten resolutions was 0.75 at $9 \mathrm{~nm}$ with two input bands. Overall, the resolution of $1 \mathrm{~nm}$ and $2 \mathrm{~nm}$ stayed relatively stable with accuracies of more than 0.60 . The ' $\mathrm{NaN}^{\prime}$ represented that there were no feature bands. 


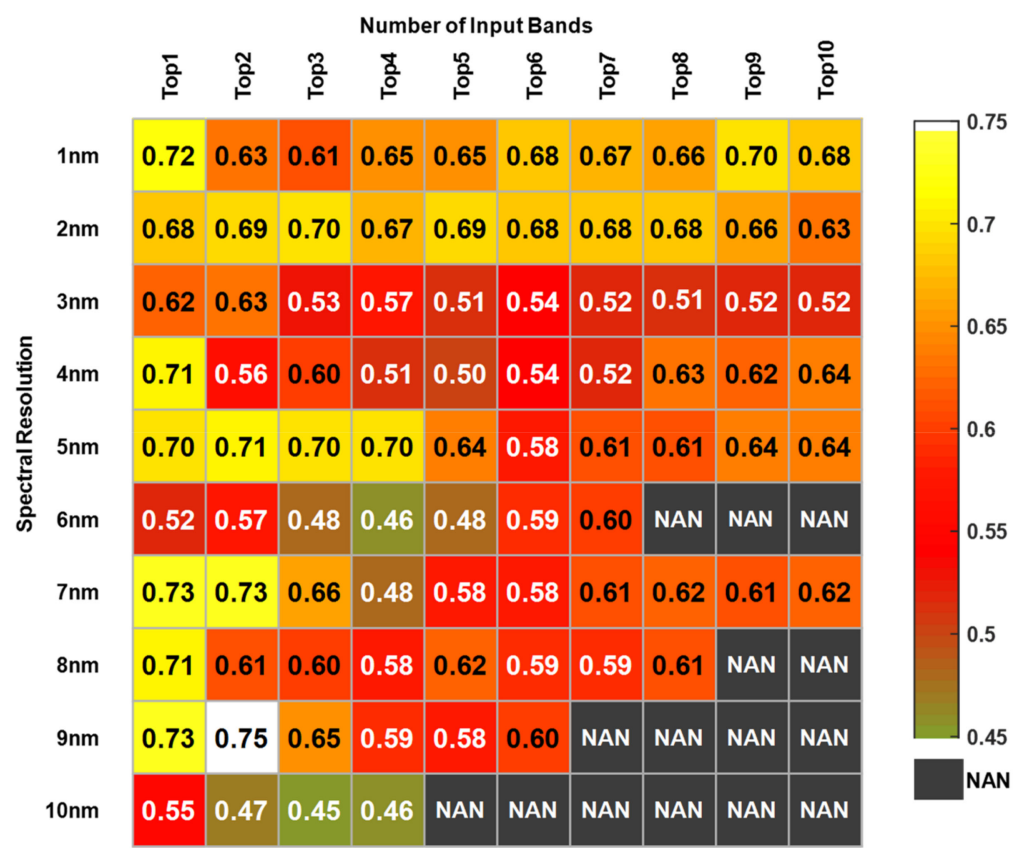

Figure 4. Diagnostic accuracies of $\mathrm{Pb}$ pollution diagnosing for band combination selecting.

By analyzing the diagnostic accuracies with the different band combinations and different resolutions, the optimal bands of each spectral resolution were determined by the highest accuracy. The details of the band combination selection for each spectral resolution were pretend in Table 2 . Primitive bands represented the number of the initial bands. ANOVA2 and RF were used to select the band combinations, successively.

Table 2. Details of the band number at each spectral resolution.

\begin{tabular}{cccccc}
\hline \multirow{2}{*}{$\begin{array}{c}\text { Spectral } \\
\text { Resolution }\end{array}$} & $\begin{array}{c}\text { Primitive } \\
\text { Bands }\end{array}$ & $\begin{array}{c}\text { Bands after } \\
\text { ANOVA2 }\end{array}$ & $\begin{array}{c}\text { Input Bands } \\
\text { after RF }\end{array}$ & $\begin{array}{c}\text { Bands after } \\
\text { ANOVA2 }\end{array}$ & $\begin{array}{c}\text { Input Bands } \\
\text { after RF }\end{array}$ \\
\cline { 3 - 6 } & 1660 & 48 & 8 & 50 & 1 \\
$2 \mathrm{~nm}$ & 830 & 34 & 4 & 31 & 3 \\
$3 \mathrm{~nm}$ & 552 & 26 & 3 & 20 & 2 \\
$4 \mathrm{~nm}$ & 415 & 28 & 7 & 11 & 1 \\
$5 \mathrm{~nm}$ & 332 & 23 & 5 & 14 & 7 \\
$6 \mathrm{~nm}$ & 275 & 21 & 2 & 7 & 1 \\
$7 \mathrm{~nm}$ & 235 & 19 & 2 & 14 & 1 \\
$8 \mathrm{~nm}$ & 207 & 17 & 5 & 8 & 2 \\
$9 \mathrm{~nm}$ & 183 & 19 & 2 & 6 & 1 \\
$10 \mathrm{~nm}$ & 166 & 12 & 7 & 4 & \\
\hline
\end{tabular}

ANOVA2 = two-way analysis of variance; and RF = random forest.

After RF selection, the number of band combinations was not more than five for most spectral resolutions. The number of sensitive feature bands of $\mathrm{Pb}$ pollution were generally less than that of the $\mathrm{Cd}$ bands. For $\mathrm{Cd}$ pollution, the number of band combinations did not exceed five in six different spectral resolutions. For Pb pollution, there was only one feature band in five different spectral resolutions. 


\subsection{Diagnostic Accuracies of Different Spectral Resolution for Different Levels}

For each resolution, the diagnostic accuracies of each pollution level also depended on the highest accuracy. After diagnosing with SVM and LOOCV validating, followed by band combination selecting, the diagnostic accuracies of different pollution levels were obtained, as shown in Table 3.

Table 3. Details of the diagnostic accuracies with different spectral resolution.

\begin{tabular}{ccccccccc}
\hline & ZCd & LCd & MCd & HCd & ZPb & LPb & $\mathbf{M P b}$ & $\mathbf{H P b}$ \\
\hline $1 \mathrm{~nm}$ & $\mathbf{0 . 7 5}$ & 0.73 & 0.69 & 0.72 & 0.69 & 0.71 & 0.73 & 0.74 \\
$2 \mathrm{~nm}$ & 0.70 & 0.72 & 0.67 & 0.69 & 0.76 & 0.65 & 0.70 & 0.68 \\
$3 \mathrm{~nm}$ & 0.72 & 0.68 & 0.73 & $\mathbf{0 . 7 5}$ & $\mathbf{0 . 8 5}$ & 0.31 & 0.72 & 0.61 \\
$4 \mathrm{~nm}$ & 0.72 & 0.72 & $\mathbf{0 . 7 4}$ & 0.66 & 0.76 & 0.64 & 0.72 & 0.73 \\
$5 \mathrm{~nm}$ & 0.55 & 0.64 & 0.68 & 0.73 & 0.79 & 0.69 & 0.68 & 0.68 \\
$6 \mathrm{~nm}$ & 0.64 & 0.61 & 0.69 & 0.70 & 0.80 & 0.41 & 0.41 & $\mathbf{0 . 7 9}$ \\
$7 \mathrm{~nm}$ & 0.69 & 0.54 & 0.66 & 0.66 & 0.77 & $\mathbf{0 . 7 3}$ & 0.73 & 0.69 \\
$8 \mathrm{~nm}$ & 0.64 & 0.58 & 0.63 & 0.54 & 0.76 & 0.63 & 0.73 & 0.73 \\
$9 \mathrm{~nm}$ & 0.61 & 0.65 & 0.71 & 0.71 & $\mathbf{0 . 8 5}$ & 0.70 & $\mathbf{0 . 7 5}$ & 0.71 \\
$10 \mathrm{~nm}$ & 0.46 & $\mathbf{0 . 7 4}$ & 0.60 & 0.71 & 0.72 & 0.64 & 0.36 & 0.47 \\
\hline
\end{tabular}

The results indicated that it was not uniform for the optimal diagnostic value of different pollution categories and levels. The highest diagnostic accuracies of the zero, low, medium, and high levels of $\mathrm{Cd}$ pollution were $0.75,0.74,0.74$, and 0.75 at $1 \mathrm{~nm}, 10 \mathrm{~nm}, 4 \mathrm{~nm}$, and $3 \mathrm{~nm}$, respectively, while the highest diagnostic accuracies for $\mathrm{Pb}$ were $0.85,0.73,0.75$, and 0.79 at $9 \mathrm{~nm}, 7 \mathrm{~nm}, 9 \mathrm{~nm}$, and $6 \mathrm{~nm}$, respectively. The highest diagnostic accuracies were above 0.70 no matter what categories and what levels the rice subject. Among all the highest diagnostic accuracies, the diagnosis of the zero-concentration $\mathrm{Pb}$ reached the optimal diagnosis with the accuracy of 0.85 .

For the Cd pollution of the zero concentration, the accuracies were not less 0.70 before the spectral resolution increased to the $5 \mathrm{~nm}$. When the spectral resolution expanded $5 \mathrm{~nm}$ or more, all accuracies were less than 0.65 except 0.69 at $7 \mathrm{~nm}$. For the low concentration diagnosing, the highest diagnostic accuracy was 0.74 at $10 \mathrm{~nm}$ resolution. In addition, the accuracies were more than 0.65 from $1 \mathrm{~nm}$ to $4 \mathrm{~nm}$. For the spectral resolution in the range from $1 \mathrm{~nm}$ to $7 \mathrm{~nm}$, the diagnostic accuracies of the medium concentration were above 0.65 , and the highest diagnostic accuracy was 0.74 at $4 \mathrm{~nm}$. Similar to the diagnosis of the zero concentration, the highest diagnostic accuracy of high concentration was also 0.75 , but the spectral resolution was different. Besides the highest diagnostic accuracy at $3 \mathrm{~nm}$, there were five resolutions with an accuracy of not less than 0.7. For the high concentration diagnosis, the accuracies were not less than 0.60 except the poorest value 0.47 at $10 \mathrm{~nm}$. The highest diagnostic accuracy was 0.79 at $6 \mathrm{~nm}$, and the accuracies exceeded 0.65 in the range from $4 \mathrm{~nm}$ to $9 \mathrm{~nm}$.

For $\mathrm{Pb}$ pollution, the highest diagnostic accuracy of zero concentration reached 0.85 , which was the highest value in all diagnosis of different concentration, and there were two spectral resolutions, $3 \mathrm{~nm}$ and $9 \mathrm{~nm}$, that all reached the highest diagnostic accuracy. Except for the spatial resolution of $1 \mathrm{~nm}$ and $3 \mathrm{~nm}$, the rest of the accuracies exceeded 0.75 . The accuracy at $1 \mathrm{~nm}$ was the lowest, but it was still close to 0.70 . For the low concentration diagnosing, the accuracy fluctuated greatly, and the resolution at $2 \mathrm{~nm}$ and $5 \mathrm{~nm}$ did not exceed 0.45 . The highest diagnostic accuracy was 0.73 at $7 \mathrm{~nm}$, while the minimum was only 0.31 at $3 \mathrm{~nm}$, and the difference was more than 0.4. Similar to the diagnosis of the low concentration, there were two resolutions that their accuracies were less than 0.45 , with the worst accuracy being only 0.36 at $10 \mathrm{~nm}$. The highest diagnostic accuracy of medium concentration was 0.75 at $9 \mathrm{~nm}$. 


\section{Discussion}

\subsection{Suitable Wavelengths Analysis for $\mathrm{Cd}-\mathrm{Pb}$ Pollution Diagnosing}

The optimal spectral resolution affects the production cost of the sensor, and the wavelengths coverage is related to the fabrication and spectral acquisition of sensor. So, we investigated the wavelength coverage of the optimal bands for each spectral resolution.

Tables 4 and 5 show the wavelengths of the input bands from $1 \mathrm{~nm}$ to $10 \mathrm{~nm}$ for single Cd and $\mathrm{Pb}$ diagnosing, and Figures 5 and 6 show the corresponding frequencies of the chosen wavelengths in all ten spectral resolutions. Due to an excess of bands, the figures only show the bands with the frequencies greater than one.

Table 4. Spectral location of the Cd pollution feature bands for any resolution.

\begin{tabular}{cl}
\hline Spectral Resolution & \multicolumn{1}{c}{ Band Width } \\
\hline $1 \mathrm{~nm}$ & $734 \mathrm{~nm}, 754-755 \mathrm{~nm}, 768-769 \mathrm{~nm}, 776 \mathrm{~nm}, 1237 \mathrm{~nm}, 1309 \mathrm{~nm}, 1831 \mathrm{~nm}$ \\
$2 \mathrm{~nm}$ & $766-773 \mathrm{~nm}, 1310-1311 \mathrm{~nm}$ \\
$3 \mathrm{~nm}$ & $719-721 \mathrm{~nm}, 752-754 \mathrm{~nm}, 767-775 \mathrm{~nm}, 818-820 \mathrm{~nm}, 836-838 \mathrm{~nm}$, \\
& $1214-1216 \mathrm{~nm}, 1310-1312 \mathrm{~nm}$ \\
$4 \mathrm{~nm}$ & $382-385 \mathrm{~nm}, 750-753 \mathrm{~nm}, 766-773 \mathrm{~nm}, 834-837 \mathrm{~nm}, 1082-1085 \mathrm{~nm}$, \\
$5 \mathrm{~nm}$ & $1298-1301 \mathrm{~nm}$ \\
$6 \mathrm{~nm}$ & $765-774 \mathrm{~nm}, 785-789 \mathrm{~nm}, 1015-1019 \mathrm{~nm}, 1080-1084 \mathrm{~nm}$ \\
$7 \mathrm{~nm}$ & $764-775 \mathrm{~nm}$ \\
$8 \mathrm{~nm}$ & $770-776 \mathrm{~nm}, 833-839 \mathrm{~nm}$ \\
$9 \mathrm{~nm}$ & $766-773 \mathrm{~nm}, 814-821 \mathrm{~nm}, 830-837 \mathrm{~nm}, 1078-1085 \mathrm{~nm}, 1222-1229 \mathrm{~nm}$ \\
$10 \mathrm{~nm}$ & $746-754 \mathrm{~nm}, 836-844 \mathrm{~nm}$ \\
& $710-719 \mathrm{~nm}, 810-819 \mathrm{~nm}, 830-839 \mathrm{~nm}, 1020-1029 \mathrm{~nm}, 1310-1319 \mathrm{~nm}$, \\
& $1340-1349 \mathrm{~nm}$ \\
\hline
\end{tabular}

Table 5. Spectral location of the $\mathrm{Pb}$ pollution feature bands for any resolution.

\begin{tabular}{cc}
\hline Spectral Resolution & Band Width \\
\hline $1 \mathrm{~nm}$ & $761 \mathrm{~nm}$ \\
$2 \mathrm{~nm}$ & $708-709 \mathrm{~nm}, 762-763 \mathrm{~nm}$ \\
$3 \mathrm{~nm}$ & $638-640 \mathrm{~nm}, 884-886 \mathrm{~nm}$ \\
$4 \mathrm{~nm}$ & $1174-1177 \mathrm{~nm}$ \\
$5 \mathrm{~nm}$ & $765-769 \mathrm{~nm}, 1891-1895 \mathrm{~nm}$ \\
$6 \mathrm{~nm}$ & $1394-1399 \mathrm{~nm}$ \\
$7 \mathrm{~nm}$ & $1771-1777 \mathrm{~nm}$ \\
$8 \mathrm{~nm}$ & $1174-1181 \mathrm{~nm}$ \\
$9 \mathrm{~nm}$ & $392-397 \mathrm{~nm}, 467-481 \mathrm{~nm}, 518-529 \mathrm{~nm}, 614-619 \mathrm{~nm}$, \\
$10 \mathrm{~nm}$ & $1178-1186 \mathrm{~nm}, 1870-1878 \mathrm{~nm}$ \\
\end{tabular}

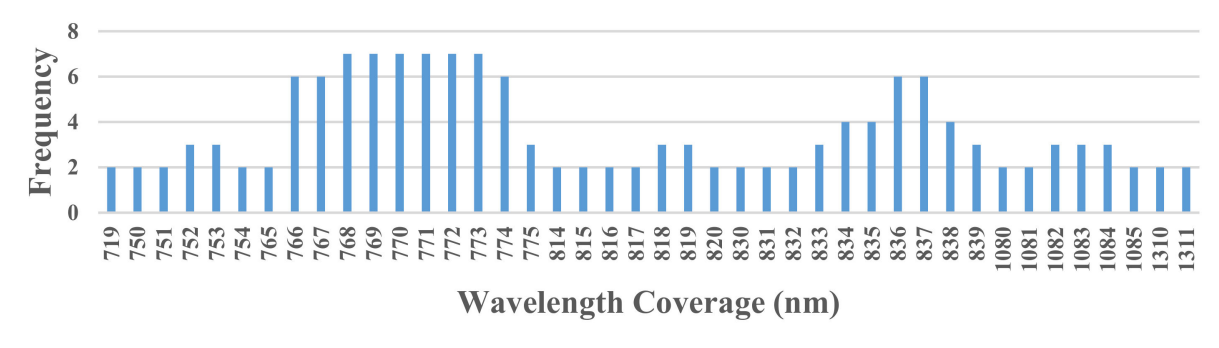

Figure 5. Frequencies of the input bands for the primitive wavelength for $\mathrm{Cd}$ pollution. 


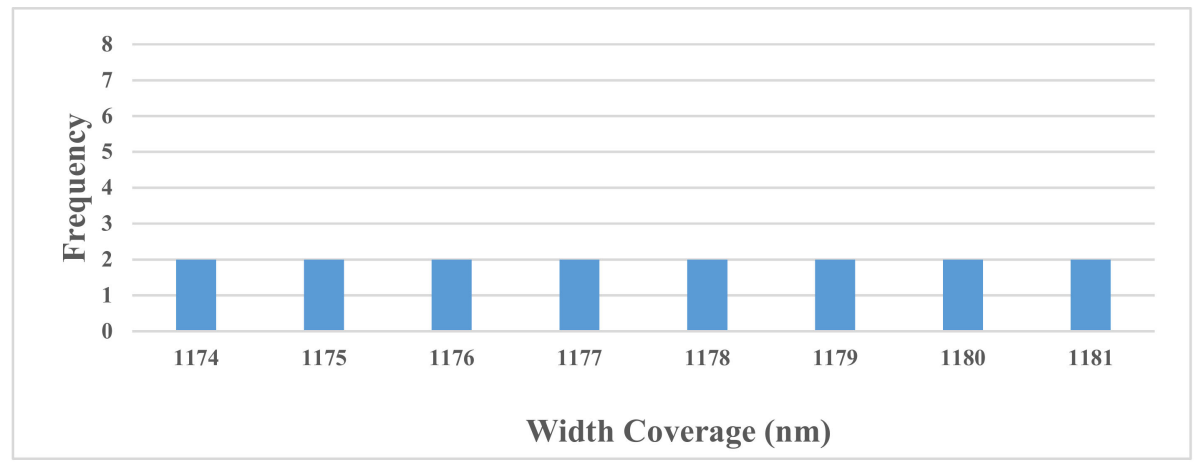

Figure 6. Frequency of the input bands of the primitive wavelength for $\mathrm{Pb}$ pollution.

As shown in Figures 5 and 6, there were forty-one bands of primitive reflectance to choose for the $\mathrm{Cd}$ pollution diagnosing, while there were only eight bands for $\mathrm{Pb}$ pollution. For $\mathrm{Cd}$ pollution diagnosing, the highest frequency was seven, including six primitive bands from $768 \mathrm{~nm}$ to $773 \mathrm{~nm}$, which were located in the near-visible wavelength coverage. Compared with the frequency of the feature bands diagnosing $\mathrm{Cd}$ pollution, the distribution position of the input feature bands with the highest frequency were relatively scattered, and the highest frequency was only two, including eight primitive reflectance bands from $1174 \mathrm{~nm}$ to $1181 \mathrm{~nm}$. Thus, subsequent research may consider focusing on $768-773 \mathrm{~nm}$ and $1174-1181 \mathrm{~nm}$ for $\mathrm{Cd}-\mathrm{Pb}$ cross contamination diagnosing.

\subsection{Optimal Spectral Resolution Analysis}

The average accuracy of the four levels was a key indicator of diagnosing ability, so the spectral resolution, depending on the highest accuracy with different spectral resolution, could be used as a reference for subsequent $\mathrm{Cd}-\mathrm{Pb}$ diagnosis and even spectrometer production. Figures 7 and 8 showed the changes of the diagnostic accuracies when only considering a single heavy metal diagnostic results, and Table 6 displayed the highest accuracies of $\mathrm{Cd}-\mathrm{Pb}$ comprehensive diagnosis after selecting band combination.
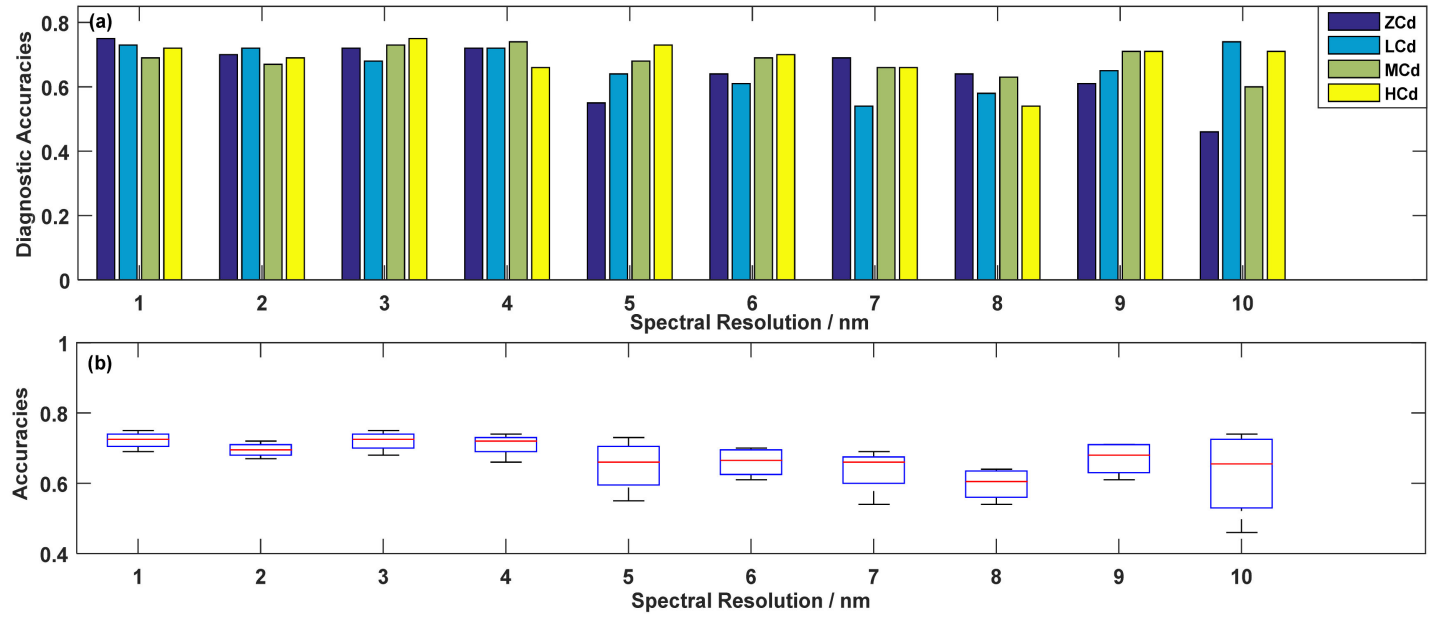

Figure 7. Optimal accuracies (a) and corresponding boxplots (b) for diagnosing Cd pollution in different resolutions.

As shown in Figure 7a, for diagnosed Cd pollution, the histograms of ZCd showed a downward trend as a whole, but there was a small ridge at the $4 \mathrm{~nm}$ resolution. The low concentration diagnostic histograms showed a trend of rising, falling, and rising again, and the accuracy at $10 \mathrm{~nm}$ was peak value. The diagnostic accuracies of the medium concentration slightly fluctuated, between 0.6 and 0.7 , and the maximum value appeared at $4 \mathrm{~nm}$. Similar to the diagnosis of the zero concentration, the 
diagnostic accuracies of the high concentration were generally decreasing, but there was a peak at $3 \mathrm{~nm}$.
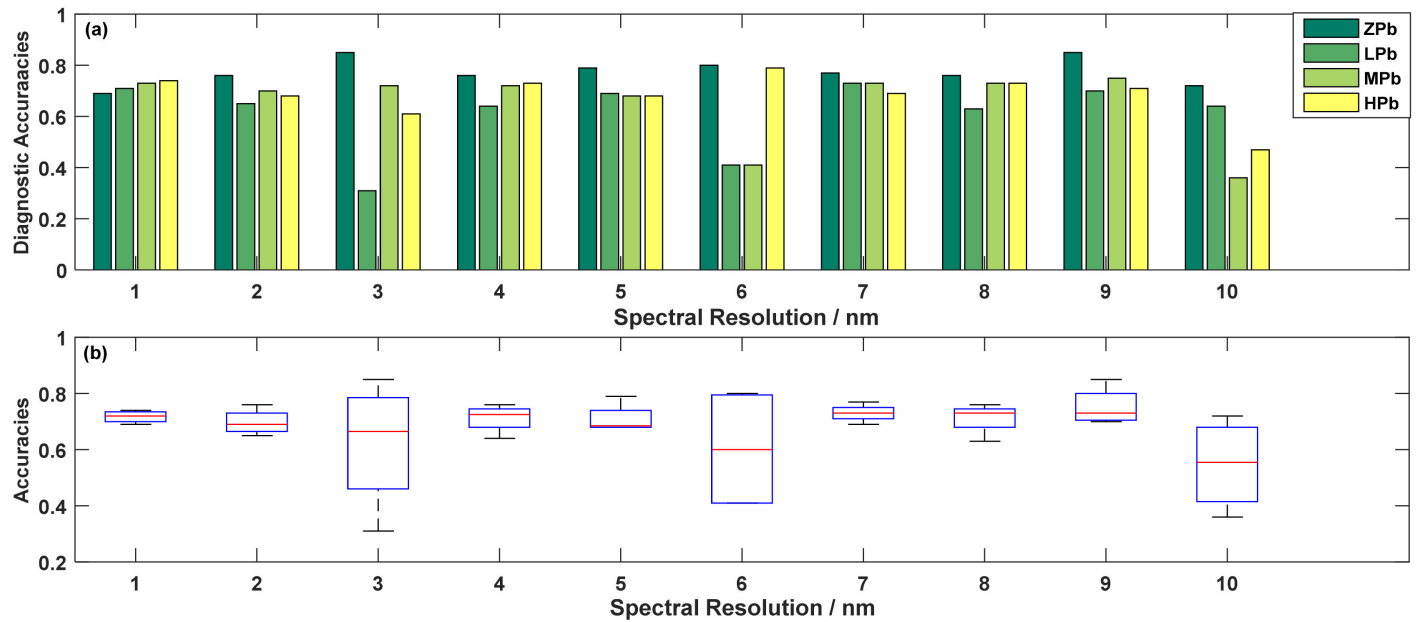

Figure 8. Optimal accuracies (a) and corresponding boxplots (b) for diagnosing $\mathrm{Pb}$ pollution in different resolutions.

Table 6. Statistical results of $\mathrm{Cd}-\mathrm{Pb}$ comprehensive diagnosis after selecting band combination.

\begin{tabular}{ccccccccccc}
\hline Spectral Resolution & $\mathbf{1} \mathbf{n m}$ & $\mathbf{2} \mathbf{~ n m}$ & $\mathbf{3} \mathbf{n m}$ & $\mathbf{4} \mathbf{n m}$ & $\mathbf{5} \mathbf{n m}$ & $\mathbf{6} \mathbf{n m}$ & $\mathbf{7} \mathbf{n m}$ & $\mathbf{8} \mathbf{n m}$ & $\mathbf{9} \mathbf{n m}$ & $\mathbf{1 0} \mathbf{n m}$ \\
\hline AV & 0.69 & 0.69 & 0.65 & 0.66 & 0.66 & 0.62 & 0.68 & 0.63 & $\mathbf{0 . 7 1}$ & 0.57 \\
Standard Deviation & 0.11 & $\mathbf{0 . 0 3}$ & 0.1 & 0.12 & 0.07 & 0.14 & 0.06 & 0.19 & $\mathbf{0 . 0 7}$ & 0.12 \\
Recall Ratio & $\mathbf{0 . 8 3}$ & 0.75 & 0.69 & 0.59 & 0.79 & 0.61 & 0.75 & 0.80 & $\mathbf{0 . 7 6}$ & 0.61 \\
Range & 0.40 & $\mathbf{0 . 1 3}$ & 0.35 & 0.43 & 0.23 & 0.41 & 0.23 & 0.55 & $\mathbf{0 . 2 4}$ & 0.35 \\
Variable Coefficient & 0.16 & $\mathbf{0 . 0 5}$ & 0.16 & 0.18 & 0.10 & 0.22 & 0.09 & 0.30 & $\mathbf{0 . 0 9}$ & 0.21 \\
\hline
\end{tabular}

The boxplots in Figure $7 \mathrm{~b}$ showed the overall diagnostic level for four different $\mathrm{Cd}$ contaminative concentrations. There are not outliers for diagnostic accuracies. The resolutions of $1 \mathrm{~nm}$ and $3 \mathrm{~nm}$ had a clear advantage with the overall better diagnostic level. The detailed accuracies at $1 \mathrm{~nm}$ were 0.75 , $0.73,0.69$, and 0.72 for zero, low, medium, and high concentration, respectively, while the accuracies at $3 \mathrm{~nm}$ were $0.72,0.68,0.73$, and 0.75 , respectively. Taking the sensors production cost into consideration, the $3 \mathrm{~nm}$ spectral resolution was a more appropriate spectral resolution for identifying whether the rice was exposed to any concentration of $\mathrm{Cd}$ pollution.

As shown in Figure 8a, for Pb pollution under a single level, the highest accuracies were generally not at $1 \mathrm{~nm}$. For the zero-concentration diagnosing, the accuracies were flat between $4 \mathrm{~nm}$ and $8 \mathrm{~nm}$, and the maximum value was 0.85 at $3 \mathrm{~nm}$ and $9 \mathrm{~nm}$. Due to the larger spectral resolution, the $9 \mathrm{~nm}$ was more suitable for diagnosing $\mathrm{ZPb}$ concentration. In the low-concentrations histograms, there was a minimum value, which indicated that it was not an appropriate resolution for $2 \mathrm{~nm}$ to diagnose the low-concentration $\mathrm{Pb}$ pollution. The medium-concentration histograms showed a stable trend in the range from $1 \mathrm{~nm}$ to $4 \mathrm{~nm}$, and then fluctuated greatly with the increase of spectral resolution. If only one pollution level was considered, the $7 \mathrm{~nm}$ was the most suitable spectral resolution for low-level $\mathrm{Pb}$ pollution diagnosing, as $9 \mathrm{~nm}$ was suitable for medium-level diagnosis. For diagnosis of the high level, there was an overall trend of falling, rising and falling again, and the accuracies reached the peak value at $6 \mathrm{~nm}$. In addition, the overall accuracy optimization might result in the poor diagnostic accuracy of a certain pollution level diagnosis. There were some lower diagnostic accuracies, such as the $\mathrm{LPb}$ diagnosis at $3 \mathrm{~nm}$ and the $\mathrm{MPb}$ diagnosis at $6 \mathrm{~nm}$, which indicated that it was not appropriate for these spectral resolutions to distinguish the $\mathrm{LPb}$ and $\mathrm{MPb}$, respectively.

It was showed for the overall diagnostic level of $\mathrm{Pb}$ contamination in Figure 8b. There were four spectral resolutions that the overall diagnostic levels were better than others, including $1 \mathrm{~nm}, 5 \mathrm{~nm}$, $7 \mathrm{~nm}$ and $9 \mathrm{~nm}$. The specific accuracies at $9 \mathrm{~nm}$ were $0.85,0.70,0.75$, and 0.71 for zero, low, medium, 
and high concentration, respectively, and the corresponding average accuracy reached 0.75 , which was the highest values in all the average accuracies. Thus, $9 \mathrm{~nm}$ was a more suitable spectral resolution for distinguishing whether rice is exposed to any concentrations of $\mathrm{Pb}$ pollution.

To find a universal spectral resolution for $\mathrm{Cd}-\mathrm{Pb}$ cross contamination diagnosing, the comprehensive accuracies of different $\mathrm{Cd}-\mathrm{Pb}$ levels should be considered. Table 6 displayed the average accuracies of $\mathrm{Cd}-\mathrm{Pb}$ comprehensive diagnosis after selecting band combination.

Except 0.57 at $10 \mathrm{~nm}$, all average accuracies overpassed 0.60. All AV values exceeded 0.65 before the spectral resolution exceeded $5 \mathrm{~nm}$. The average accuracy reached 0.71 at $9 \mathrm{~nm}$, which was also the highest value in all average accuracies. To further confirm the reliability of the highest accuracy, we also calculated the standard deviation, the recall rate, range (xmax-xmin), and variable coefficient of diagnostic accuracies at different resolutions.

The higher value of recall ratio represented the stronger diagnostic ability for pre-diagnostic concentrations, while the smaller value of standard deviation (SD), ranges, and variable coefficient represented the better stability in different concentrations. The calculated results showed that the diagnostic accuracy at $9 \mathrm{~nm}$ had a relatively small standard deviation of 0.07 and a relatively high recall rate of 0.76 . In addition, the range and variable coefficient in $9 \mathrm{~nm}$ also kept a more stable condition relatively with a value of 0.24 and 0.09 respectively. Although there was a better stability in the resolution of $2 \mathrm{~nm}$, the resolution of $9 \mathrm{~nm}$ was higher in AV and recall ration with a good stability. Therefore, the $9 \mathrm{~nm}$ was optimal spectral resolution for $\mathrm{Cd}-\mathrm{Pb}$ cross contamination diagnosing.

In this study, the diagnostic accuracies by high-resolution measurement were not always more accurate than low-resolutions ones, for example, the diagnostic accuracies of $C d$ contamination at $8 \mathrm{~nm}$ did not outperform the $9 \mathrm{~nm}$ ones. The effect might be related to the Hughes phenomenon [23] and the collinearity between hyperspectral bands $[20,21]$. On the one hand, the spectral averaging eliminated random errors, caused by the working state of the machine in the process of spectral acquisition. On the other hand, some adjacent collinear bands with high sensitivity may be selected preferentially in the process of selecting feature bands, which may cause the loss of spectral information $[22,36,37]$. As a result, higher accuracies might be obtained in low resolution. In addition, Marceau's research results [38] showed that the spectral variability within the category was also a cause.

Based on the above results and discussions, we propose that $9 \mathrm{~nm}$ is the optimal spectral resolution to sensor production for $\mathrm{Cd}-\mathrm{Pb}$ cross contamination diagnosing simultaneously in rice, and give a suggestion that spectral resolutions of $3 \mathrm{~nm}$ and $9 \mathrm{~nm}$ should be the optimal resolutions for diagnosing the single $\mathrm{Cd}$ and $\mathrm{Pb}$ pollution. The sampling interval of $1.4 \mathrm{~nm}$, like ASD FieldSpec®3, may be appropriate due to the closed relationship between the sensitive bands' wavelength coverage and short-wave infrared range. Using the optimal spectral resolution may improve diagnostic stability and reduce the cost of the instrument.

\section{Conclusions}

This paper proposed an optimal spectral resolution for diagnosing the categories and levels of $\mathrm{Cd}-\mathrm{Pb}$ cross contamination in rice based on the hyperspectral dataset. ANOVA2 and RF were used to select the feature bands, followed by SVM and cross validation to get diagnostic accuracies. By analyzing diagnostic results of different band combinations and different spectral resolution, the band combinations and the optimal spectral resolutions, ranging from $1 \mathrm{~nm}$ to $10 \mathrm{~nm}$, were chosen based on the highest accuracy. The results indicated that: (1) the hyperspectral technology was a promising method for diagnosing heavy metal cross contamination of $\mathrm{Cd}$ and $\mathrm{Pb}$. For each spectral resolution, no matter what categories and levels of $\mathrm{Cd}$ and $\mathrm{Pb}$ rice suffered, the first-rank average diagnostic accuracies were above 0.6 , except for $\mathrm{Pb}$ pollution diagnosing with the $10 \mathrm{~nm}$ resolution. (2) Wavelengths of 768-773 $\mathrm{nm}$ and 1174-1181 nm might be worth exploring to find a suitable index for distinguishing the $\mathrm{Cd}$ and $\mathrm{Pb}$ pollution. (3) The $9 \mathrm{~nm}$ was the optimal spectral resolution to instruct the sensor production for $\mathrm{Cd}-\mathrm{Pb}$ cross contamination diagnosing. 
This experiment only investigated the optimal spectral resolution diagnosing the $\mathrm{Cd}-\mathrm{Pb}$ cross contamination. It is worthy of exploring the feasibility of the proposed method to determine the optimal spectral resolution of other heavy metals cross contamination in the future research. This future exploration may find a universal resolution diagnosing heavy-metal pollutions, which can help expand the diagnostic scopes of the sensor.

Author Contributions: S.Z. designed and performed the experiments, and write original draft preparation; T.F. made an investigation and conceived the experiment; Y.Z. and T.F. reviewed and edited the paper; M.W. made the project administration and funding acquisition.

Funding: This work was supported by the National Natural Science Foundation of China (grant numbers 41801382, 91838303).

Acknowledgments: The authors would like to thank Yiyun Chen for his suggestions in structure of the paper and his support in software, thank Yanli Wang, Luxiao He for their help in charts making, and thank the handling editor and three anonymous reviewers for their detailed and constructive comments and suggestions, which greatly helped inn improving the quality of this paper.

Conflicts of Interest: The authors declare no conflicts of interest.

\section{References}

1. Khan, S.; Cao, Q.; Zheng, Y.M.; Huang, Y.Z.; Zhu, Y.G. Health risks of heavy metals in contaminated soils and food crops irrigated with wastewater in Beijing, China. Environ. Pollut. 2008, 152, 686-692. [CrossRef] [PubMed]

2. Salazar, M.J.; Rodriguez, J.H.; Leonardo Nieto, G.; Pignata, M.L. Effects of heavy metal concentrations (Cd, $\mathrm{Zn}$ and $\mathrm{Pb}$ ) in agricultural soils near different emission sources on quality, accumulation and food safety in soybean [Glycine max (L.) Merrill]. J. Hazard. Mater. 2012, 233, 244-253. [CrossRef] [PubMed]

3. Leenaers, H.; Okx, J.P.; Burrough, P.A. Employing elevation data for efficient mapping of soil pollution on floodplains. Soil Use Manag. 1990, 6, 105-114.

4. Steiger, B.V.; Webster, R.; Schulin, R.; Lehmann, R. Mapping heavy metals in polluted soil by disjunctive kriging. Environ. Pollut. 1996, 94, 205-215. [CrossRef]

5. Huang, W.; Davidw, L.; Zheng, N.; Zhang, Y.; Liu, L.; Wang, J. Identification of yellow rust in wheat using in-situ spectral reflectance measurements and airborne hyperspectral imaging. Precis. Agric. 2007, 8, 187-197. [CrossRef]

6. Wang, D.; Wilson, C.; Shannon, M.C. Interpretation of salinity and irrigation effects on soybean canopy reflectance in visible and near-infrared spectrum domain. Int. J. Remote Sens. 2002, 23, 811-824. [CrossRef]

7. Rud, R.; Shoshany, M.; Alchanatis, V. Spectral indicators for salinity effects in crops: a comparison of a new green indigo ratio with existing indices. Remote Sens. Lett. 2010, 2, 289-298. [CrossRef]

8. Shi, T.; Chen, Y.; Liu, Y.; Wu, G. Visible and near-infrared reflectance spectroscopy-an alternative for monitoring soil contamination by heavy metals. J. Hazard. Mater. 2014, 265, 166-176. [CrossRef] [PubMed]

9. Liu, Y.; Li, W.; Wu, G.; Xu, X. Feasibility of estimating heavy metal contaminations in floodplain soils using laboratory-based hyperspectral data—A case study along Le'an River, China. Geosp. Inf. Sci. 2011, 14, 10-16. [CrossRef]

10. Chen, T.; Chang, Q.; Clevers, J.G.; Kooistra, L. Rapid identification of soil cadmium pollution risk at regional scale based on visible and near-infrared spectroscopy. Environ. Pollut. 2015, 206, 217-226. [CrossRef] [PubMed]

11. Shi, T.; Wang, J.; Chen, Y.; Wu, G. Improving the prediction of arsenic contents in agricultural soils by combining the reflectance spectroscopy of soils and rice plants. Int. J. Appl. Earth Obs. Geoinf. 2016, 52, 95-103. [CrossRef]

12. Kooistra, L.; Wehrens, R.; Leuven, R.S.; Buydens, L.M. Possibilities of visible-near-infrared spectroscopy for the assessment of soil contamination in river floodplains. Anal. Chim. Acta 2001, 446, 97-105. [CrossRef]

13. Malley, D.F.; Williams, P.C. Use of Near-Infrared Reflectance Spectroscopy in Prediction of Heavy Metals in Freshwater Sediment by Their Association with Organic Matter. Environ. Sci. Technol. 1997, 31, 3461-3467. [CrossRef] 
14. Moshou, D.; Bravoa, C.; Westb, J.; Wahlena, S.; Mccartneyb, A.; Ramona, H. Automatic Detection Of 'Yellow Rust' In Wheat Using Reflectance Measurements And Neural Networks. Comput. Electron. Agric. 2004, 44, 173-188. [CrossRef]

15. Tilley, D.R.; Ahmed, M.; Son, J.H.; Badrinarayanan, H. Hyperspectral reflectance response of freshwater macrophytes to salinity in a brackish subtropical marsh. J. Environ. Qual. 2007, 36, 780-789. [CrossRef] [PubMed]

16. St. Luce, M.; Ziadi, N.; Gagnon, B.; Karam, A. Visible near infrared reflectance spectroscopy prediction of soil heavy metal concentrations in paper mill biosolid- and liming by-product-amended agricultural soils. Geoderma 2017, 288, 23-36.

17. Choe, E.; van der Meer, F.; van Ruitenbeek, F.; van der Werff, H.; de Smeth, B.; Kim, K.-W. Mapping of heavy metal pollution in stream sediments using combined geochemistry, field spectroscopy, and hyperspectral remote sensing: A case study of the Rodalquilar mining area, SE Spain. J. Environ. Qual. 2008, 112, 3222-3233. [CrossRef]

18. Wang, J.; Cui, L.; Gao, W.; Shi, T.; Chen, Y.; Gao, Y. Prediction of low heavy metal concentrations in agricultural soils using visible and near-infrared reflectance spectroscopy. Geoderma 2014, 216, 1-9. [CrossRef]

19. Liu, M.; Liu, X.; Ding, W.; Wu, L. Monitoring stress levels on rice with heavy metal pollution from hyperspectral reflectance data using wavelet-fractal analysis. Int. J. Appl. Earth Obs. Geoinf. 2011, 13, $246-255$. [CrossRef]

20. Vandermeulen, R.A.; Mannino, A.; Neeley, A.; Werdell, J.; Arnone, R. Determining the optimal spectral sampling frequency and uncertainty thresholds for hyperspectral remote sensing of ocean color. Opt. Express 2017, 25, A785-A797. [CrossRef]

21. Nidamanuri, R.R.; Zbell, B. A method for selecting optimal spectral resolution and comparison metric for material mapping by spectral library search. Prog. Phys. Geog. 2010, 34, 47-58. [CrossRef]

22. Dalponte, M.; Bruzzone, L.; Vescovo, L.; Gianelle, D. The role of spectral resolution and classifier complexity in the analysis of hyperspectral images of forest areas. Remote Sens. Environ. 2009, 113, 2345-2355. [CrossRef]

23. Hughes, G.P. On the mean accuracy of statistical pattern recognizers. IEEE Trans. Inf. Theory 1968, 14, 55-63. [CrossRef]

24. Fu, J.; Zhou, Q.; Liu, J.; Liu, W.; Wang, T.; Zhang, Q.; Jiang, G. High levels of heavy metals in rice (Oryza sativa L.) from a typical E-waste recycling area in southeast China and its potential risk to human health. Chemosphere 2008, 71, 1269-1275. [PubMed]

25. Gao, W.; Whiting, M.L.; Jackson, T.J. Measuring surface water in soil with light reflectance. Int. Soc. Opt. Photonics 2009, 7454, 74540D.

26. MEE; SAMR. Risk Control Standard for Soil Contamination of Agricultural Land; GB15618-2018; Standards Press of China Beijing: Beijing, China, 2018; pp. 1-10. (In Chinese)

27. Jiang, Q.; Liu, M.; Wang, J.; Liu, F. Feasibility of using visible and near-infrared reflectance spectroscopy to monitor heavy metal contaminants in urban lake sediment. Catena 2018, 162, 72-79. [CrossRef]

28. Kooistra, L.; Salas, E.A.L.; Clevers, J.G.P.W.; Wehrens, R.; Leuven, R.S.E.W.; Nienhuis, P.H.; Buydens, L.M.C. Exploring field vegetation reflectance as an indicator of soil contamination in river floodplains. Environ. Pollut. 2004, 127, 281-290. [CrossRef]

29. Kemper, T.; Ehlers, M.; Sommer, S.; Posa, F.; Kaufmann, H.J.; Michel, U.; De Carolis, G. Use of airborne hyperspectral data to estimate residual heavy metal contamination and acidification potential in the Guadiamar floodplain Andalusia, Spain after the Aznacollar mining accident. Int. Soc. Opt. Photonics 2004, $5574,224-234$.

30. Wang, T.; Wei, H.; Zhou, C.; Gu, Y.; Li, R.; Chen, H.; Ma, W. Estimating cadmium concentration in the edible part of Capsicum annuum using hyperspectral models. Environ. Monit. Assess. 2017, 189, 548. [CrossRef]

31. Sridhar, B.B.M.; Vincent, R.K.; Roberts, S.J.; Czajkowski, K. Remote sensing of soybean stress as an indicator of chemical concentration of biosolid amended surface soils. Int. J. Appl. Earth Obs. Geoinf. 2011, 13, 676-681. [CrossRef]

32. Maanan, M.; Saddik, M.; Maanan, M.; Chaibi, M.; Assobhei, O.; Zourarah, B. Environmental and ecological risk assessment of heavy metals in sediments of Nador lagoon, Morocco. Ecol. Indic. 2015, 48, 616-626. [CrossRef] 
33. Jin, X.; Du, J.; Liu, H.; Wang, Z.; Song, K. Remote estimation of soil organic matter content in the Sanjiang Plain, Northest China: The optimal band algorithm versus the GRA-ANN model. Agric. For. Meteorol. 2016, 218, 250-260. [CrossRef]

34. Martens, H.E.; Naes, T. Multivariate Calibration. Biometrics 1989, 47, 380-395.

35. Savitzky, A.; Golay, M.J.E. Smoothing and Differentiation of Data by Simplified Least Squares Procedures. Anal. Chem. 1964, 36, 1627-1639. [CrossRef]

36. Wang, D.; Liu, X. Comparative Analysis of GF-1 and HJ-1 Data to Derive the Optimal Scale for Monitoring Heavy Metal Stress in Rice. Int. J. Environ. Res. Public Health 2018, 15, 461. [CrossRef] [PubMed]

37. Lu, H.; Yu, X.; Zhou, L.; He, Y. Selection of Spectral Resolution and Scanning Speed for Detecting Green Jujubes Chilling Injury Based on Hyperspectral Reflectance Imaging. Appl. Sci. 2018, 8, 523. [CrossRef]

38. Marceau, D.J.; Hay, G.J. Remote Sensing Contributions to the Scale Issue. Can. J. Remote Sens. 1999, 25, 357-366. [CrossRef]

(C) 2019 by the authors. Licensee MDPI, Basel, Switzerland. This article is an open access article distributed under the terms and conditions of the Creative Commons Attribution (CC BY) license (http://creativecommons.org/licenses/by/4.0/). 